\title{
Zukunftsträchtige Technik
}

Der Kongress der Deutschen Gesellschaft für Phoniatrie und Pädaudiologie

(DGPP) fand vom 21. - 23. September in Bonn statt. Das Hauptthema:

technische Entwicklungen in der Phoniatrie und Pädaudiologie.

\section{Spezielle Programmierung des Cochlea-Implantats lindert Tinnitus}

D atienten mit höhergradiger Schwerhörigkeit berichten häufig über Tinnitus verschiedener Schweregrade. Auch nach einer Cochlea-Implantat-Versorgung ist noch jeder zehnte Patient betroffen. In vielen Fällen nimmt der Tinnitus nach Versorgung mit einem Cochlea-Implantat (CI) zwar ab, bei manchen Patienten jedoch nur vorübergehend. Das bestätigt eine Untersuchung einer Arbeitsgruppe um Dr. Frank Engel vom Bundeswehrzentralkrankenhaus Koblenz bei 55 Patienten mit CI-Versorgung: Vor Implantation litten 24\% (13 Patienten) unter mittlerem bis schwerem Tinnitus, nach Implantation noch $11 \%$ (sechs Patienten).

Um Tinnitus bei CI-Patienten besser behandeln zu können, wurden bei den

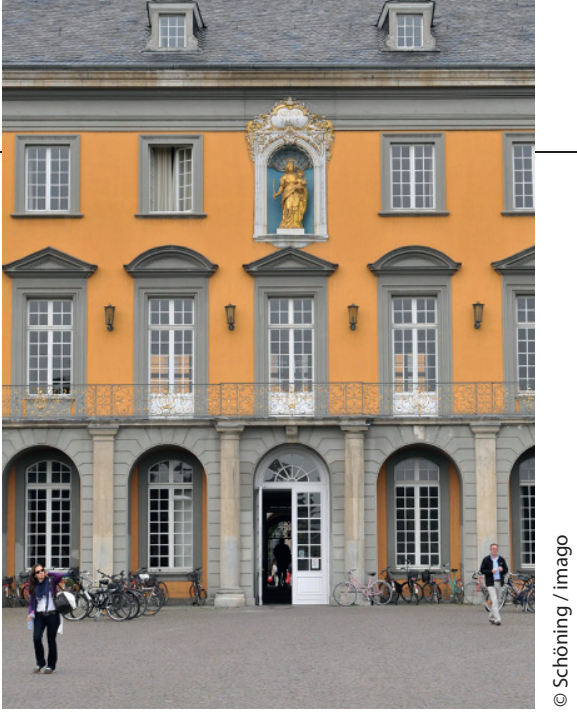

sechs betroffenen Patienten spezielle Programmierstrategien des CI erprobt: das Anheben der Lautheit aller Frequenzen, das Anheben des Niederfrequenzbereichs und das Anheben des Hochfrequenzbereichs. Als erfolgversprechendster Ansatz habe sich das Anheben der apikalen Lautheiten erwiesen, berichtete Engel.

Engel F: Prävalenz, Diagnostik und Therapie von Tinnitus bei Cochlear-ImplantPatienten

\section{Narrow Band Imaging in der Larynxdiagnostik}

n der Früherkennung des Larynxkarzinoms spielt die richtige Einschätzung verdächtiger Schleimhautläsionen durch den Untersucher eine wesentliche Rolle. Um die diagnostische Genauigkeit zu verbessern, wurden in den vergangenen Jahren verschiedene endoskopische bildgebende Verfahren entwickelt. Dazu zählt auch das Narrow Band Imaging (NBI). Die Technik basiert auf dem Einsatz von schmalbandigen Farbfiltern, die den Rotanteil im Farbspektrum reduzieren und das blaue Lichtspektrum betonen. Die erzeugten Wellenlängen werden vor allem von Hämoglobin resorbiert und verbessern die Kontrastierung mikrovaskulärer Strukturen in der Mukosa

Weitere Infos auf springermedizin.de

Weitere Berichte zu interessanten Themen des Kongresses finden Sie auf springermedizin.de

-www.springermedizin.de/ dgpp-kongress-2012 und Submukosa. Blaulicht mit einer Frequenz zwischen 400 und $430 \mathrm{~nm}$ dient der Darstellung von oberflächlichen Kapillaren in der Schleimhaut, während grünes Licht mit einer Frequenz zwischen 525 und $555 \mathrm{~nm}$ submuköse Gefäße visualisiert.

Prospektive Studien belegen, dass sich mittels NBI sowohl die Detektion maligner Schleimhautläsionen verbessern lässt als auch die Beurteilung papillomatöser Läsionen der laryngealen Schleimhaut.

Eine Arbeitsgruppe um Dr. Katrin Jahn von der Universitäts-HNO-Klinik Bonn konnte ein flexibles NBI-Laryngoskop bei 21 Patienten in der Praxis testen. Einbezogen wurden Patienten mit verschiedenen Schleimhautläsionen im Bereich des Nasopharynx und Larynx. Die Untersuchungen wurden tansnasal unter Oberflächenanästhesie durchgeführt. Ein einfacher Wechsel zwischen verschiedenen Belichtungsoptionen per Knopfdruck am Endoskop erlaubte einen direkten Vergleich von Befunden in konventionellem Weißlicht mit einer Darstellung im NBI-Modus.

Das NBI-Verfahren erwies sich dabei als wertvolles Instrument für die laryngologische Praxis, berichtete Jahn und erläu- terte dies an einigen Fallbeispielen: So konnte bei einem Patienten mit Larynxpapillomatose die charakteristische blumenkohlartige Oberfläche der Papillome mit den typischen punktförmigen Läsionen im NBI deutlicher dargestellt werden als in konventionellem Weißlicht; zudem wurde ein beginnender Befall der vorderen Kommissur und der Stimmlippenschleimhaut der Gegenseite deutlicher. Bei einem Rezidiv einer Stimmlippenleukoplakie waren durch die Kontrastierung im NBI-Modus die Begrenzungen der Läsion besser zu erkennen, zusätzlich zeigte sich ein schmaler Ausläufer nach dorsal, der unter Weißlicht nicht sichtbar war. Bei einem Stimmlippenkarzinom sowie bei einem Plattenepithelkarzinom ließen sich die Tumorgrenzen klarer darstellen als mittels konventionellem Weißlicht.

Das NBI könne somit die diagnostische Genauigkeit bei Schleimhautläsionen wesentlich verbessern, schlussfolgerte Jahn.

Jahn K: Erste Erfahrungen mit dem Narrow Band Imaging (NBI) in der laryngologischen Praxis.

Berichterstattung: Angelika Bauer-Delto 